\title{
Outcomes of Stent-Assisted Coiling Using the Neuroform Atlas Stent in Unruptured Wide-Necked Intracranial Aneurysms
}

\author{
Ohyuk Kwon, Joonho Chung \\ Department of Neurosurgery, Severance Hospital, Yonsei University College of Medicine, Seoul, Korea
}

Objective : Although stent-assisted coiling (SAC) has been reported to be safe and effective in treating wide-necked aneurysms, the technique has procedure-related complications. Thus, we reported our experiences of SAC using the Neuroform Atlas stent in treating wide-necked aneurysms and evaluated the incidence of and risk factors for procedure-related complications.

Methods : From March 2018 to August 2019, we treated 130 unruptured wide-necked aneurysms in 123 patients with Neuroform Atlas stents. Angiographic results and clinical outcomes were reviewed retrospectively. Clinical and angiographic follow-up were performed in all cases (mean, 12.4 months) after the procedure.

Results : There were eight cases (6.2\%) of procedure-related complications (two dissections, five thromboembolisms, and one hemorrhage) and two (1.5\%) of delayed complications (one ischemia and one hemorrhage). There was one case (0.8\%) of failure of stent deployment and one (0.8\%) of suboptimal positioning of the stent. Follow-up angiography showed complete obliteration in 103 (79.2\%), residual neck in 16 (12.3\%), and residual aneurysm in 11 cases (8.5\%). Aneurysm locations in the middle cerebral artery (odds ratio $[\mathrm{OR}], 2.211 ; p=0.046)$ and the anterior communicating artery $(\mathrm{OR}, 2.850 ; p=0.039)$ were associated with procedurerelated complications on univariate analysis. However, no independent risk factor for procedure-related complications was noted in multivariate analysis.

Conclusion : The Neuroform Atlas showed a high rate of technical success. Good clinical and radiographic outcomes in early follow-up suggests that the device is feasible and safe. SAC of aneurysms on the middle cerebral artery or anterior communicating artery may require more attention to prevent possible procedure-related complications.

Key Words : Endovascular procedures $\cdot$ Intracranial aneurysm $\cdot$ Stents.

\section{INTRODUCTION}

It is well established that wide-necked intracranial aneurysms are difficult to treat endovascularly due to the risk of coil protrusion in the parent artery and aneurysm recurrence. Stent-assisted coiling (SAC) has been established as one of the treatment options for these endovascularly challenging aneurysms, thereby improving the results. Intracranial stents allow the reconstruction of the aneurysm neck and help maintain the coil mass within the aneurysm sac. Recently, the Neuroform Atlas stent (Stryker Neurovascular, Kalamazoo, MI, USA), a microcatheter-delivered, self-expanding, mixed open-

- Received : February 27, 2020 •Revised : March 18, 2020 •Accepted : March 30, 2020

- Address for reprints : Joonho Chung

Department of Neurosurgery, Severance Hospital, Yonsei University College of Medicine, 50-1 Yonsei-ro, Seodaemun-gu, Seoul 03722, Korea

Tel : +82-2-2228-2150, Fax : +82-2-393-9979, E-mail : ns.joonho.chung@gmail.com, ORCID : https://orcid.org/0000-0003-2745-446X

This is an Open Access article distributed under the terms of the Creative Commons Attribution Non-Commercial License (http://creativecommons.org/licenses/by-nc/4.0) which permits unrestricted non-commercial use, distribution, and reproduction in any medium, provided the original work is properly cited. 
cell/closed-cell stent, has been used widely. With low-profiled, easy handling deliveries, the Neuroform Atlas stent is being widely applied for wide-necked intracranial aneurysms in various ways $^{9,12)}$.

Although SAC using the Neuroform Atlas stent has been reported to be safe and effective in treating wide-necked aneurysms ${ }^{1,6,7,13,14)}$, the technique has procedure-related complications that interventionists need to understand completely. However, none of those studies reported the incidence of and risk factors for procedure-related complications. Thus, this study aimed to report our experience with SAC using the Neuroform Atlas stent in treating unruptured wide-necked aneurysms and to evaluate the incidence of and risk factors for procedure-related complications.

\section{MATERIALS AND METHODS}

\section{Patient selection}

This retrospective study was approved by Yonsei University Health System, Severance Hospital, Institutional Review Board (4-2020-0573), and the requirement for informed consent was waived. The Neuroform Atlas was approved for use in our country in March 2018. From March 2018 to August 2019, we treated 435 aneurysms (367 unruptured and 68 ruptured) in 381 patients endovascularly. Among 214 aneurysms treated with SAC, 130 unruptured wide-necked aneurysms in 123 patients were treated with Neuroform Atlas stents. Among the 68 ruptured aneurysms cases during the same period, we tried to perform endovascular treatment other than the use of stents considering our results pertaining to SAC for ruptured aneurysms ${ }^{3)}$.

Aneurysm size and morphological characteristics were determined using digital subtraction angiography with 3D rotational imaging. A wide-necked saccular aneurysm was defined as that with a dome-to-neck ratio of $<2$ or a neck diameter of $\geq 4 \mathrm{~mm}$. Thromboembolic complications were defined as angiographic evidence of thrombosis during or at the end of SAC. Asymptomatic post-operative small diffusion restrictions on diffusion weighted image were not regarded as thromboembolic complications. Hemorrhagic complications were defined as newly developed hemorrhage on computed tomography (CT) or magnetic resonance image within 30 days of the procedure. The incidence of and risk factors for procedure-related complica- tions were retrospectively evaluated.

\section{Endovascular treatment}

The treatment decision was made based on agreement between microvascular neurosurgeons and neuro-interventionists. All SAC procedures were performed under general anesthesia. A 5-Fr to 7-Fr guiding catheter was positioned in the internal carotid artery for anterior circulation aneurysms. A 5 -Fr or 6-Fr guiding catheter was positioned in the vertebral artery for posterior circulation aneurysms. Intravenous systemic heparin $(50 \mathrm{U} / \mathrm{kg}$ ) was administered while placing the guiding catheter. An activated clotting time of 2-2.5 times that of baseline was maintained during endovascular treatment. An Excelsior SL-10 (Stryker Neurovascular) was used to deliver the Neuroform Atlas stent. The jailing or semi-jailing techniques were primarily performed, and if these failed, the through the strut (cell-through) technique was implemented.

Patients were administered a daily dose of clopidogrel $75 \mathrm{mg}$ and aspirin $100 \mathrm{mg}$ for more than 7 days before the SAC procedure. For patients with insufficient premedication or when the SAC was planned immediately after the diagnostic digital subtraction angiography, a loading dose of aspirin and clopidogrel of $300 \mathrm{mg}$ each was administered the day before treatment. Platelet function testing was routinely performed for all patients. After the procedure, patients were prescribed $75 \mathrm{mg}$ of clopidogrel daily for 3 months and $100 \mathrm{mg}$ of aspirin daily for at least 12 months. Magnetic resonance imaging was routinely performed within 24 hours after SAC for all patients and included diffusion-weighted imaging, T2-weighted imaging, fluid-attenuated inversion recovery, and gradient echo imaging.

\section{Clinical and radiographic outcomes}

Angiographic results and clinical outcomes were reviewed retrospectively. Clinical and angiographic follow-up was performed in all 123 patients with 130 aneurysms between 3 and 20 months (mean, 12.4 months) after the procedure. The angiographic results were evaluated by two independent investigators and categorized as complete occlusion (without a neck remnant), neck remnant (>90\% occlusion with small neck remnant or dog ear), or partial occlusion (filling of the aneurysm lumen). Clinical outcomes were assessed using the modified Rankin Scale and evaluated at the time of discharge and out-patient follow-up by an independent investigator who was 
not involved in patient care.

\section{Statistical analysis}

All statistical analyses were performed after consulting with a biostatistician using $\mathrm{R}$ language ver. 3.01 (R Foundation for Statistical Computing, Vienna, Austria). Student's t-tests were used for numeric variables. Chi-square tests were used for nominal variables. Logistic regression analysis was performed to determine the independent risk factors for procedure-related complications. Multiple logistic regression analyses were performed for variables with an unadjusted effect and with a $p$ value $<0.10$ in simple logistic regression analysis. The 95\% confidence interval (CI) was calculated for all $p$ values, which were considered statistically significant at $<0.05$.

\section{RESULTS}

Baseline demographics and aneurysm characteristics are summarized in Table 1. Of the 123 patients included in this

Table 1. Baseline demographics and aneurysm characteristics

\begin{tabular}{lc}
\hline & Value \\
\hline Patients & 123 \\
Age (years) & $60.2 \pm 10.1$ \\
Female & $96(78.0)$ \\
Hypertension & $63(51.2)$ \\
Diabetes & $29(23.6)$ \\
Smoking & $19(15.4)$ \\
Dyslipidemia & $16(13.0)$ \\
Coronary heart disease & $11(8.9)$ \\
Aneurysm & 130 \\
Aneurysm size (mm) & $5.3 \pm 1.7$ \\
Neck size (mm) & $3.9 \pm 0.8$ \\
Aneurysm location & \\
Internal carotid artery & $65(50.0)$ \\
Posterior communicating artery & $9(6.9)$ \\
Middle cerebral artery & $18(13.8)$ \\
Anterior communicating artery & $17(13.1)$ \\
Anterior cerebral artery & $10(7.7)$ \\
Basilar artery & $9(6.9)$ \\
Vertebral artery & $2(1.5)$ \\
\hline
\end{tabular}

Values are presented as mean \pm standard deviation or number (\%) study, there were 96 women, and the median age was 60.2 years (range from 29 to 79). Patient baseline characteristics included hypertension in 63 patients (51.2\%), diabetes in 29 (23.6\%), smoking history in 19 (15.4\%), dyslipidemia in 16 (13.0\%), and coronary heart diseases in 11 (8.9\%). Aneurysms were located in the internal carotid artery in 65 cases (50.0\%), posterior communicating artery in nine (6.9\%), middle cerebral artery in 18 (13.8\%), anterior communicating artery in 17 (13.1\%), anterior cerebral artery in 10 (7.7\%), basilar artery in nine (6.9\%), and vertebral artery in two (1.5\%). The mean aneurysm size was $5.3 \pm 1.7 \mathrm{~mm}$, and the mean neck size was $3.9 \pm$ $0.8 \mathrm{~mm}$.

Table 2 shows the clinical and radiographic outcomes of the aneurysms. In terms of technical considerations, there was one case $(0.8 \%)$ of failure of stent deployment and one $(0.8 \%)$ of suboptimal positioning of the stent. These two events hap-

Table 2. Clinical and radiographic outcomes of the aneurysms

\begin{tabular}{lc}
\hline & Value $(\mathbf{n}=\mathbf{1 3 0})$ \\
\hline Technical considerations & $1(0.8)$ \\
Deployment failure & $1(0.8)$ \\
Suboptimal positioning & $3(2.3)$ \\
Y-stenting & $6(4.6)$ \\
Stent salvage & \\
Initial angiographic results & $61(46.9)$ \\
Complete obliteration & $44(33.8)$ \\
Residual neck & $25(19.2)$ \\
Residual aneurysm & \\
Follow-up angiographic results & $103(79.2)$ \\
Complete obliteration & $16(12.3)$ \\
Residual neck & $11(8.5)$ \\
Residual aneurysm & $8(6.2)$ \\
Procedure-related complications & $2(1.5)$ \\
Dissection & $5(3.8)$ \\
Thromboembolism & $1(0.8)$ \\
Hemorrhage & $2(1.5)$ \\
Delayed complications & $1(0.8)$ \\
Ischemia & $1(0.8)$ \\
Memorrhage & $0(0.0)$ \\
\hline Morbidity (mRS 1-5) & $0(0.0)$ \\
\hline Sicality & \\
\hline
\end{tabular}

mRS : modified Rankin Scale 
pened because the delivery microcatheters suddenly dropped to the proximal part of very tortuous internal carotid arteries. We performed Y-stenting (Atlas-Atlas; Stryker Neurovascular, Fremont, CA, USA) in three patients (2.3\%) and stent salvage in six (4.6\%). Immediate postembolization angiographic results showed complete obliteration in 61 aneurysms (46.9\%), residual neck in 44 (33.8\%), and residual aneurysm in 25 (19.2\%). Follow-up angiographic results were obtained in all aneurysms and showed complete obliteration in 103 (79.2\%), residual neck in 16 (12.3\%), and residual aneurysm in 11 (8.5\%). There was no recurrence of the aneurysm during the follow-up period. With respect to clinical outcomes, there were no morbidity or mortality cases even though there were eight cases (6.2\%) of procedure-related complications (two dissections, five thromboembolisms, and one hemorrhage) and two (1.5\%) of delayed complications (one ischemia and one hemorrhage). All five patients with thromboembolic complications underwent intra-arterial thrombolysis in which a glycoprotein IIb/IIIa antagonist (tirofiban) was used and showed successful recanalization without any symptoms. Two patients experienced vertebral artery dissection due to guiding catheter placement and were treated conservatively.

Logistic regression analysis was used to determine independent associations of procedure-related complications with other factors. On univariate analysis, aneurysm locations in the middle cerebral artery (odds ratio [OR], 2.211; 95\% CI, 1.196-3.993;

Table 3. Potential risk factors for procedure-related complications

\begin{tabular}{|c|c|c|c|c|c|c|}
\hline \multirow[t]{2}{*}{ Variable } & \multicolumn{2}{|c|}{$\begin{array}{l}\text { Procedure-related } \\
\text { complications }\end{array}$} & \multicolumn{2}{|c|}{ Unadjusted } & \multicolumn{2}{|l|}{ Adjusted } \\
\hline & Yes $(n=8)$ & No $(n=122)$ & OR $(95 \% \mathrm{CI})$ & $p$-value & OR $(95 \% \mathrm{Cl})$ & $p$-value \\
\hline Age (years) & $59.0 \pm 10.4$ & $60.3 \pm 9.9$ & $1.094(0.944-1.326)$ & 0.823 & $0.975(0.962-1.149)$ & 0.652 \\
\hline \multicolumn{7}{|l|}{ Sex } \\
\hline Female $(n=102)$ & $6(75.0)$ & $96(78.7)$ & 1 & & & \\
\hline Male $(n=28)$ & $2(25.0)$ & $26(21.3)$ & $1.085(0.517-2.023)$ & 0.575 & $1.029(0.554-1.898)$ & 0.711 \\
\hline Hypertension, yes & $6(75.0)$ & $62(50.8)$ & $2.616(0.828-9.205)$ & 0.138 & & \\
\hline Diabetes, yes & $2(25.0)$ & $27(22.1)$ & $1.312(0.750-3.206)$ & 0.676 & & \\
\hline Smoking, yes & $1(12.5)$ & $18(14.8)$ & $0.816(0.471-3.054)$ & 0.765 & & \\
\hline Dyslipidemia, yes & $0(0.0)$ & $16(13.1)$ & $0.625(0.433-5.224)$ & 0.218 & & \\
\hline Coronary heart disease, yes & $0(0.0)$ & $11(8.5)$ & $0.687(0.682-3.469)$ & 0.270 & & \\
\hline \multicolumn{7}{|l|}{ Aneurysm location } \\
\hline Internal carotid artery $(n=65)$ & $1(12.5)$ & $64(52.5)$ & 1 & & & \\
\hline Posterior communicating artery $(n=9)$ & $0(0.0)$ & $9(7.4)$ & $0.972(0.300-2.832)$ & 0.442 & & \\
\hline Middle cerebral artery $(n=18)$ & $3(37.5)$ & $15(12.3)$ & $2.211(1.196-3.993)$ & 0.046 & $1.525(0.943-3.021)$ & 0.088 \\
\hline Anterior communicating artery $(n=17)$ & $3(37.5)$ & $14(11.5)$ & $2.850(1.298-5.143)$ & 0.039 & $2.559(0.954-4.622)$ & 0.067 \\
\hline Anterior cerebral artery $(n=10)$ & $0(0.0)$ & $10(8.2)$ & $0.575(0.447-1.866)$ & 0.385 & & \\
\hline Basilar artery $(n=9)$ & $1(12.5)$ & $8(6.6)$ & $0.727(0.486-1.978)$ & 0.524 & & \\
\hline Vertebral artery $(n=2)$ & $0(0.0)$ & $2(1.6)$ & $0.922(0.907-1.054)$ & 0.810 & & \\
\hline \multicolumn{7}{|l|}{ Aneurysm neck } \\
\hline Relative wide neck (<4 mm, n=76) & $6(75.0)$ & $70(57.4)$ & 1 & & & \\
\hline Absolute wide neck ( $\geq 4$ mm, $n=54$ ) & $2(25.0)$ & $52(42.6)$ & $0.922(0.668-3.218)$ & 0.186 & & \\
\hline \multicolumn{7}{|l|}{ Aneurysm size } \\
\hline Small $(<5 \mathrm{~mm}, \mathrm{n}=64)$ & $5(62.5)$ & $59(48.4)$ & 1 & & & \\
\hline Medium $(<10 \mathrm{~mm}, \mathrm{n}=59)$ & $3(37.5)$ & $56(45.9)$ & $0.750(0.638-2.169)$ & 0.302 & & \\
\hline Large $(\geq 10 \mathrm{~mm}, \mathrm{n}=7$ ) & $0(0.0)$ & $7(5.7)$ & $0.332(0.305-1.811)$ & 0.489 & & \\
\hline
\end{tabular}

Values are presented as mean \pm standard deviation or number (\%) unless otherwise indicated. OR : odds ratio, $\mathrm{Cl}$ : confidence interval 
$p=0.046)$ and the anterior communicating artery (OR, 2.850; 95\% CI, 1.298-5.143; $p=0.039$ ) was associated with procedurerelated complications (Table 3 ). However, no independent risk factor for procedure-related complications was noted in the multivariate logistic regression analysis adjusted for age and sex.

\section{DISCUSSION}

In the present study, the procedure-related complication rate was $6.2 \%$ among 123 patients with 130 unruptured widenecked aneurysms. Further, no case of morbidity/mortality was noted with SAC for unruptured wide-necked intracranial aneurysms with the Neuroform Atlas. Thromboembolic complications were the most common at 3.8\% followed by dissection $(1.5 \%)$ and hemorrhage $(0.8 \%)$. The technical success rate of delivering and deploying the stent was 98.5\% among the 130 cases. Delayed ischemia due to transient ischemic attack and a small amount of delayed hemorrhage in the left frontal lobe occurred in one case each during the follow-up period, with both patients being asymptomatic. We found that stent deployment in the middle cerebral artery and anterior communicating artery was associated with procedure-related complications in the univariate analysis.

An expected benefit of the Neuroform Atlas stent is its increased navigability, which allows easy delivery ${ }^{12}$. The Neuroform Atlas stent is the latest version of the Neuroform series with reinforced navigability within a smaller microcatheter that confers stability to the vessel wall (high radial force), enables accurate placement (ease of use with very low foreshortening), and allows wall apposition, conformability, and coil protection. It can be delivered via a 0.0165 -inch microcatheter, the smallest profile, allowing neurointerventionists to perform SAC without difficulty. The cell size of the Neuroform Atlas stent is smaller compared to that of its predecessor, the Neuroform stent. This enables better coil protection in aneurysms and allows the use of smaller coils. Additionally, the width of the struts has been reduced to improve flexibility and conformability of the stent. Furthermore, the segmental opening of the struts allows stable positioning and direct vessel wall apposition by anchoring the stent after the first rows of the struts exit the microcatheter. These characteristics of the Neuroform Atlas make SAC safer and more effective.

There have been a few reports on SAC with Neuroform At- las stents, of which most have reported that SAC using Neuroform Atlas stents is safe and effective in treating wide-necked aneurysms $^{1,6,7,13,14)}$. From their experience with 27 consecutive patients, the authors of one study concluded that Neuroform Atlas SAC is feasible for treating ruptured and unruptured wide-necked aneurysms that are not amenable to conventional coiling or clipping ${ }^{13)}$. The aneurysm occlusion and favorable clinical outcomes were consistent with those previously reported for SAC of wide-neck aneurysms using other devices ${ }^{13)}$. These previous authors reported favorable and complete occlusion rates of $69.2 \%$ and $53.8 \%$ at 6 months, respectively. Another report concluded that deployment of the Neuroform Atlas stent was a safe and effective method for the treatment of wide-necked aneurysms from their experiences with 37 aneurysms in 36 patients $^{14)}$. They reported satisfactory immediate angiographic outcomes : complete aneurysm obliteration was observed in $84 \%$ cases, while neck remnants were observed in the remaining 16\%. Furthermore, high-resolution time-of-flight magnetic resonance angiography revealed an occlusion rate of $100 \%$ at the 5-month follow-up. Additionally, other reports showed a complete occlusion rate of $86.7 \%$ with the Neuroform Atlas system ${ }^{6}$. The complete occlusion rate of $79.2 \%$ in the present study is in accordance with the results published previously, highlighting the efficacy of the Neuroform Atlas.

Compared to the Low-profile Visualized Intraluminal Support Junior device (LVIS Jr; MicroVention, Tustin, CA, USA), the Neuroform Atlas is less visible because only the stent markers at each end of the stent are radiopaque, although the struts themselves are invisible. Nevertheless, the visibility of the Neuroform Atlas was sufficient to allow accurate placement and deployment of the device in the present study. Additionally, the high outward radial force of the Neuroform Atlas ensures opening and wall apposition of the stent. If the complete opening of the stent is doubtful, the struts can be visualized by unenhanced flat-panel detector $\mathrm{CT}^{13)}$. This approach can be employed to overcome the disadvantage of the open-cell design of the Neuroform Atlas stent, which causes resheathing after its deployment difficult. However, neurointerventionists should be aware of the possibility of delivery microcatheters unexpectedly falling down in tortuous parent arteries for the optimal deployment of the stent. According to the thromboembolic complications, it seems that there is no difference in the rate of thromboembolic complications be- 
tween Neuroform Atlas and LVIS stents. We reported 6.5\% of thromboembolic complications using Neuroform Atlas stents, whereas LVIS family (LVIS and LVIS Jr) had 3.6-5.8\%,2,5,15). However, intra-procedural thromboembolic complications occurred in 8\% among 25 patients in SAC of anterior communicating artery aneurysms using the LVIS Jr stent ${ }^{10)}$, supporting our result of possible higher rate of procedure-related complications in the parent arteries with small diameter, such as middle cerebral artery or anterior communicating artery. Another report showed that a series of 100 patients treated with the LVIS Jr stent with thromboembolic complications occurring in $14 \%$ of the cases and concluded that the potentially inadequate antiplatelet response and use of the stent itself are possible reasons for thromboembolic phenomena in patients with acutely ruptured aneurysms ${ }^{11)}$.

With increasing clinical experience and development of new devices, SAC of aneurysms with a small parent artery has been known to be feasible in selected cases and show good long-term patency rates of parent arteries ${ }^{4,8,16,17)}$. Regardless of the stent type (open-cell or closed-cell), the deployment of stents in small arteries, such as anterior cerebral arteries, middle cerebral arteries, posterior cerebral arteries, or posterior inferior cerebellar arteries, was safe and resulted in good patency. Nevertheless, the possibility of thromboembolic complications and parent artery occlusion should not be neglected when performing SAC of aneurysms with a small parent artery. In the present study, no independent risk factors for procedure-related complications could be determined through a multivariate logistic regression analysis adjusted for age and sex. However, SAC of aneurysms located in the middle cerebral artery or anterior communicating artery showed an association with procedure-related complications in univariate analysis. Thus, using the Neuroform Atlas in distal small parent arteries might be feasible, although its possible procedurerelated complications should be considered.

Stents with an open-cell design show better wall apposition in tortuous vessels compared with closed-cell stents. However, incomplete stent apposition is more likely to be found in small arteries with tortuous anatomy. In addition, the low radial force of stents could be one cause of poor wall apposition. Although poor wall apposition may improve by open-cell stents, it might not work properly in a small-sized tortuous parent artery. The stent might not fully deploy in small arteries with acute angles because of kinking, twisting, or ovalization at the angles. This is especially concerning in arteries with a small diameter. Furthermore, increased metal surface coverage might increase the risk of thromboembolism when stents are deployed in small arteries. Thus, it can't be too careful of performing SAC in the parent artery with small diameter, such as anterior cerebral artery or middle cerebral artery.

The present study had several limitations including the retrospective data collection and the small number of patients with an unruptured aneurysm enrolled, and thus, the effects of possible selection bias cannot be excluded. Moreover, as the follow-up period was short (less than 2 years), longer followup is needed to assess the stability of adequate obliteration. Furthermore, there was no true control group for the evaluation of the efficacy and safety of the device. Thus, comparisons with different devices and laboratory experiments to improve the technical defects of the Neuroform Atlas should be performed in additional clinical trials.

\section{CONCLUSION}

The Neuroform Atlas stent showed a high rate of technical success with good navigability. Good clinical and radiographic outcomes in early follow-up suggest that the device is safe and reliable with minimal risks for SAC of unruptured intracranial aneurysms in both anterior and posterior circulation. In cases of SAC of unruptured intracranial aneurysms in the middle cerebral artery or anterior communicating artery, more careful use of the Neuroform Atlas may be required to prevent possible procedure-related complications. Large cohort studies with a longer follow-up are necessary to determine the long-term durability and safety of the device as well as the risk factors for procedure-related complications.

\section{CONFLICTS OF INTEREST}

No potential conflict of interest relevant to this article was reported.

\section{INFORMED CONSENT}

This type of study does not require informed consent. 


\section{AUTHOR CONTRIBUTIONS}

\author{
Conceptualization : JC \\ Data curation : OK, JC \\ Formal analysis : $\mathrm{OK}$ \\ Methodology: OK, JC \\ Writing - original draft : OK \\ Writing - review \& editing : JC
}

\section{ORCID}
Ohyuk Kwon
https://orcid.org/0000-0002-9065-1055
Joonho Chung
https://orcid.org/0000-0003-2745-446X

\section{References}

1. Caragliano AA, Papa R, Pitrone A, Limbucci N, Nappini S, Ruggiero $M_{t}$ et al. : The low-profile Neuroform Atlas stent in the treatment of widenecked intracranial aneurysms - immediate and midterm results: an Italian multicenter registry. J Neuroradiol, 2019 [Epub ahead of print]

2. Cho YD, Sohn CH, Kang HS, Kim JE, Cho WS, Hwang G, et al. : Coil embolization of intracranial saccular aneurysms using the Low-profile Visualized Intraluminal Support (LVISTM) device. Neuroradiology 56 : 543-551, 2014

3. Chung J, Lim YC, Suh SH, Shim YS, Kim YB, Joo JY, et al. : Stent-assisted coil embolization of ruptured wide-necked aneurysms in the acute period: incidence of and risk factors for periprocedural complications. J Neurosurg $121:$ 4-11, 2014

4. Chung J, Suh SH, Hong CK, Joo JY, Lim YC, Shin YS, et al. : Preliminary experience with self-expanding closed-cell stent placement in small arteries less than $2 \mathrm{~mm}$ in diameter for the treatment of intracranial aneurysms. J Neurosurg 122 : 1503-1510, 2015

5. Iosif C, Piotin M, Saleme S, Barreau X, Sedat J, Chau Y, et al. : Safety and effectiveness of the Low Profile Visualized Intraluminal Support (LVIS and LVIS Jr) devices in the endovascular treatment of intracranial aneurysms: results of the TRAlL multicenter observational study. J Neurointerv Surg $10: 675-681,2018$
6. Jankowitz BT, Hanel R, Jadhav AP, Loy DN, Frei D, Siddiqui $A H$, et al. : Neuroform Atlas Stent System for the treatment of intracranial aneurysm: primary results of the Atlas Humanitarian Device Exemption cohort. J Neurointerv Surg $11:$ 801-806, 2019

7. Kim CH, Kim YH, Sung SK, Son DW, Song GS, Lee SW : Clinical safety and effectiveness of stent-assisted coil embolization with Neuroform Atlas stent in intracranial aneurysm. J Korean Neurosurg Soc $63: 80$ 88,2020

8. Kühn AL, Hou SY, Puri AS, Silva CF, Gounis MJ, Wakhloo AK : Stentassisted coil embolization of aneurysms with small parent vessels: safety and efficacy analysis. J Neurointerv Surg 8 : 581-585, 2016

9. Park KY, Jang CK, Lee JW, Kim DJ, Kim BM, Chung J : Preliminary experience of stent-assisted coiling of wide-necked intracranial aneurysms with a single microcatheter. BMC Neurol $19: 245,2019$

10. Santillan A, Schwarz J, Boddu S, Gobin YP, Knopman J, Patsalides A : Stent-assisted coil embolization of anterior communicating artery aneurysms using the LVIS Jr stent. Interv Neuroradiol 25 : 12-20, 2019

11. Shankar JJS, Quateen A, Weill A, Tampieri D, Del Pilar Cortes M, Fahed $R$, et al. : Canadian registry of LVIS Jr for treatment of intracranial aneurysms (CaRLA). J Neurointerv Surg 9 : 849-853, 2017

12. Shim YS, Park SK, Chung J : Preliminary experience of stent salvage using Neuroform Atlas stent for procedure-related complication during coil embolization of intracranial aneurysms. Clin Neurol Neurosurg 190 : 105654, 2020

13. Ten Brinck MFM, de Vries J, Bartels RHMA, Grotenhuis JA, Boogaarts $H D$ : Neuroform Atlas stent-assisted coiling: preliminary results. Neurosurgery 84 : 179-189, 2019

14. Ulfert C, Pham M, Sonnberger M, Amaya F, Trenkler J, Bendszus M, et al. : The Neuroform Atlas stent to assist coil embolization of intracranial aneurysms: a multicentre experience. J Neurointerv Surg 10 : 11921196, 2018

15. Yoo DH, Cho YD, Moon J, Lee J, Kang HS, Cho WS, et al. : Long-term outcomes of Low-profile Visualized Intraluminal Support device usage in stent-assisted coiling of intracranial aneurysm. J Clin Neurosci 50 : 287-291, 2018

16. Yun JH, Cho CS : Experiences of Neuroform stent applications for ruptured anterior communicating artery aneurysms with small parent vessel. J Korean Neurosurg Soc 48 : 53-58, 2010

17. Zhang J, Lv X, Jiang C, Li Y, Yang X, Wu Z : Endovascular treatment of cerebral aneurysms with the use of stents in small cerebral vessels. Neurol Res 32 : 119-122, 2010 